\title{
Effects of early postnatal growth restriction and subsequent catch-up growth on body composition, insulin sensitivity, and behavior in neonatal rats
}

Ming-Yu Jou' ', Bo Lönnerdal' and lan J. Griffin²

BACKGROUND: Early postnatal growth retardation with subsequent catch-up growth is common in preterm infants. We describe a model of ex utero (postnatal) growth retardation followed by varying degrees of catch-up growth in the neonatal rat. METHODS: Newborn CD rat pups were randomized to litters of 10 (NN, normal then normal intake) or 16 ( $R$, restricted intake). On day 10, R pups were further randomized to litters of 6 ( $R C$, restricted then catch-up intake), 10 ( $R N$, restricted then normal intake), or 16 (RR, restricted then restricted intake). Body weight, body composition, insulin sensitivity, biochemistry, and learning (passive avoidance test) were assessed.

RESULTS: Growth was significantly lower in the $\mathrm{R}$ than the NN group. Subsequently, the RC group caught up with the NN group but had higher fat mass; the RN group showed partial catch-up but body composition similar to that of the NN group. Insulin sensitivity did not differ between groups. Learning behavior was significantly better in the NN than the three R groups, and in the RC group than the RR or RN groups. CONCLUSION: Early postnatal growth retardation is associated with poorer medium-term growth and poorer developmental outcome. Increased catch-up growth is associated with improved developmental outcome but with increased body adiposity, without any significant effect on glucose homeostasis.

$\mathbf{P}$ ostnatal growth failure is common in preterm infants $(1,2)$. As a group, preterm infants are born near the mean for in utero (fetal) growth but lose weight initially and then grow more slowly than the in utero rate (1). In one study, weight $\mathrm{z}$-scores of preterm infants ( $<30 \mathrm{wk}$ gestation) fell by 1.14 between birth and hospital discharge (2). More immature infants do even more poorly, with those born at 24-29 wk gestation being $60-70 \%$ of their expected weight by discharge (1). After hospital discharge, there is a variable amount of catch-up growth, but overall, preterm infants remain smaller than their term peers (3) and more than half remain below the 10th percentile of weight for age at 18-22 mo of age (4).

Early growth failure in preterm infants is known to have significant long-term effects. At 18-22 mo, preterm infants with the lowest rates of in-hospital weight gain are at the highest risk for Bayley's psychomotor development index and motor development index scores less than 70 , as well as for cerebral palsy, neurodevelopmental impairment, and readmission to hospital (4). Subsequent catch-up in head circumference is associated with improved long-term neurodevelopmental outcome (5), but the effect of subsequent weight catch-up is less clear. In one study on very low birth weight (VLBW) infants, failure for weight to catch up by $2 \mathrm{y}$ of age was associated with poorer neurodevelopment (6).

The growth pattern of preterm infants (underweight at term, with variable degrees of catch-up) is superficially similar to that of the intrauterine growth-retarded fetuses and has raised the concern that catch-up growth may put them at increased risk of metabolic syndrome, as is known to occur in intrauterine growth-retarded fetuses (7). However, there are a number of important differences; in intrauterine growth-retarded fetuses, the nutritional insult and poor growth occur prenatally, when carbohydrate is the dominant metabolic fuel; in the preterm infants, the insult occurs postnatally, when fat is a more important substrate. Furthermore, catch-up growth in intrauterine growth-retarded fetuses leads to overweight, whereas weight typically remains below average in the preterm infant despite catch-up growth. There is little evidence that catch-up growth in preterm infants increases their risk of metabolic syndrome or reduces insulin sensitivity, although there are some data suggesting that less weight loss during the first $2 \mathrm{wk}$ of life may increase measures of insulin resistance in adolescents born preterm (8).

Because prenatal and postnatal nutritional insults may not be comparable, it is unclear how current animal models of in utero growth retardation apply to the ex utero growth retardation of human preterm infants. The objectives of this study were to establish a rodent model of early postnatal growth retardation, occurring at an age equivalent to the third trimester for human preterm infants, followed by varying degrees of postnatal catch-up growth, and to examine the effect of these different growth patterns on body size, body composition, developmental outcome, and biochemical measures of glucose 
homeostasis (insulin sensitivity/resistance). We hypothesized that early postnatal growth retardation would lead to changes in these outcomes, which would be modified by the degree of subsequent catch-up growth.

\section{RESULTS}

\section{Growth}

On day 2, animals were randomized among 7 litters of 10 pups each ( 5 male and 5 female, normal intake $(\mathrm{N}))$ and 14 litters of 16 pups each ( 8 male and 8 female, restricted intake (R)). The R group was slightly, but significantly, heavier than the $\mathrm{N}$ group at randomization; however, the $\mathrm{N}$ group was significantly heavier at every other time point (Figure 1). By day 10 , the R group pups weighed $\sim 20 \%$ less than the $\mathrm{N}$ group pups.

On day $10, \mathrm{~N}$ pups continued in litters of 10 each $(\mathrm{NN}$, normal then normal intake, $n=4$ litters), but animals from the $\mathrm{R}$ group were rerandomized to litters of 6 (RC, restricted then catch-up intake, $n=5$ litters), 10 pups per litter (RN, restricted then normal intake, $n=4$ litters), or 16 pups per litter (RR, restricted then restricted intake, $n=4$ litters). By chance, there were small but statistically significant differences in the RC, RN, and RR groups on day 10. By day 16, the RC group had caught up with the $\mathrm{NN}$ group, and the weights of the two groups were statistically indistinguishable; the RR group showed no catchup growth, and the RN group showed partial catch-up growth. Between days 16 and 22, the four groups ran parallel to each other, and there was no further catch-up growth (Figure 1).

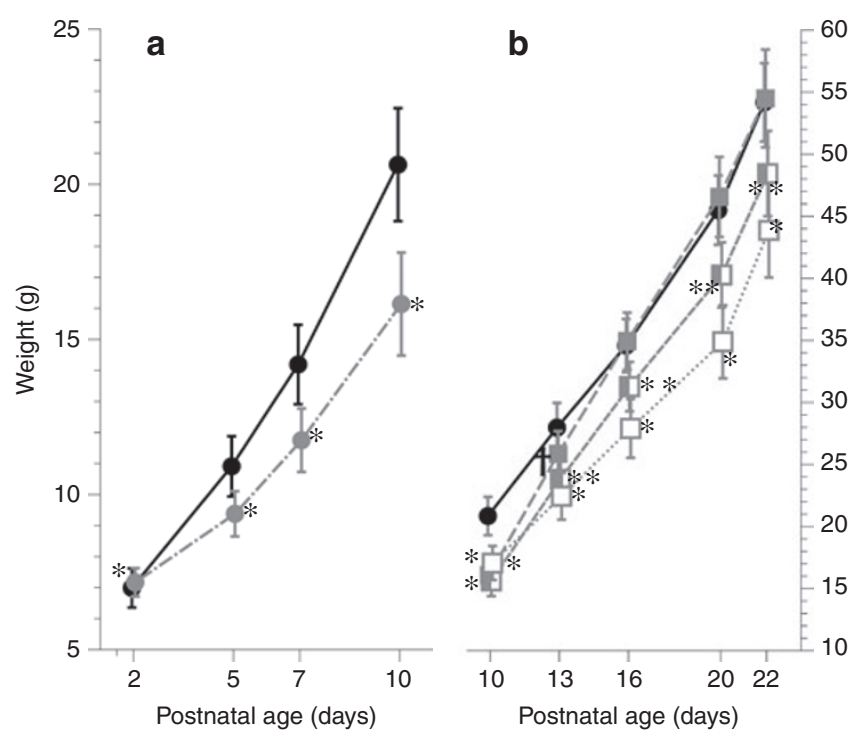

Figure 1. Weight (g) of the (a) normal ( $\mathrm{N}$, solid black circle, black line) and restricted ( $R$, solid gray circles and broken line) intake groups between days 2 and 10 and (b) between the NN (normal then normal intake, solid black circle, black line), RR (restricted then restricted intake, open white boxes and broken line), RN (restricted then normal intake, solid gray boxes and broken line), and RC (restricted then catch-up intake, half-grey halfwhite boxes and broken line) groups between days 10 and 22. In a, ${ }^{*}$ significantly different from $\mathrm{N}(P<0.05)$. In $\mathbf{b}$, * significantly different from NN $(P<0.05)$; ** significantly different from NN $(P<0.05)$ and $\operatorname{RR}(P<0.05)$; and ${ }^{\dagger}$ significantly different from NN $(P<0.05), \mathrm{RC}(P<0.05)$, and $\mathrm{RR}(P<0.05)$.
On day 22, pups from the RR group weighed $20 \%$ less than the NN pups; the RN group pups weighed $10 \%$ less than the $\mathrm{NN}$ group pups; and the $\mathrm{NN}$ and RC groups were statistically indistinguishable.

\section{Body Composition}

On day 22, NN animals consisted of 70.9\% water (SD: 0.98), $18.5 \%$ protein (SD: 0.47 ), $7.7 \%$ fat (SD: 1.07 ), and $2.9 \%$ ash (SD: 0.05). RR animals had significantly higher body water and significantly lower fat than the NN animals (Figure 2). By contrast, RC animals had significantly lower total body water, significantly lower protein mass, and significantly higher fat mass than the NN animals. There were no differences in body composition between the $\mathrm{NN}$ and $\mathrm{RN}$ animals.

\section{Biochemical Analyses}

On day 10, whole-blood glucose was significantly lower in the $\mathrm{R}$ animals than in the $\mathrm{N}$ animals (121 \pm 14 vs. $136 \pm 18 \mathrm{mg} / \mathrm{dl}$; $P<0.001)$.

On day 22, fasting blood glucose was unaffected by group or by gender $(P=0.14)$ even when current weight was accounted for $(P=0.21$; Table 1$)$. Serum insulin, homeostatic model assessment-insulin resistance, and quantitative insulin sensitivity check index were not significantly different among the groups on day 22 (Table 1 ).

Serum insulin-like growth factor-1 (IGF-1) was significantly $(P=0.0308)$ affected by group when gender was accounted for (Table 2, Model 1). However, when current weight was also accounted for (Table 2, Model 2), group had no effect on IGF-1 $(P=0.51)$, although weight was highly significant $(P<0.001)$. Serum leptin was significantly different among the groups whether (Model 1, $P=0.003$ ) or not (Model 2, $P<$ 0.0001 ) weight was accounted for, and serum leptin in the RC group was significantly higher than that in any other groups

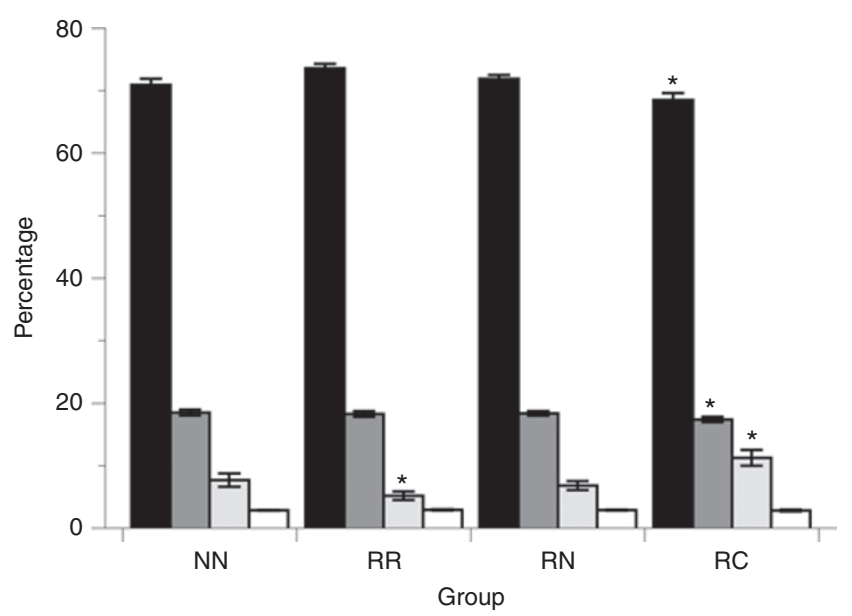

Figure 2. Percentage of water (black bars), protein (dark gray bars), fat (pale gray bars), and ash (white bars) for the four study groups on day 22. Error bars represent \pm 1 SD. Bars marked with an asterisk are significantly different from the corresponding bar in the NN group. NN, normal then normal intake; $\mathrm{RC}$, restricted then catch-up intake; $\mathrm{RN}$, restricted then normal intake; RR, restricted then restricted intake. 


\section{Articles | Jouet al.}

Table 1. Measures of glucose homeostasis (fasting glucose, insulin, HOMA-IR, HOMA- $\beta$, and QUICKI) in the four groups

\begin{tabular}{|c|c|c|c|c|c|c|}
\hline Group & NN & $\mathrm{RC}$ & $\mathrm{RN}$ & $\mathrm{RR}$ & Model 1 ( $P$ value) & Model 2 ( $P$ value) \\
\hline Fasting glucose (mg/dl) & $161 \pm 19$ & $158 \pm 25$ & $164 \pm 17$ & $152 \pm 17$ & 0.14 & 0.21 \\
\hline Serum insulin & $1.02 \pm 0.46$ & $1.01 \pm 0.34$ & $0.89 \pm 0.43$ & $0.85 \pm 0.43$ & 0.57 & 0.45 \\
\hline HOMA- $\beta$ & $4.08 \pm 2.90$ & $4.20 \pm 2.12$ & $3.32 \pm 2.13$ & $3.43 \pm 1.51$ & 0.61 & 0.34 \\
\hline QUICKI & $0.46 \pm 0.038$ & $0.46 \pm 0.400$ & $0.48 \pm 0.055$ & $0.49 \pm 0.060$ & 0.17 & 0.16 \\
\hline
\end{tabular}

HOMA- $\beta$, homeostatic model assessment- $\beta$; HOMA-IR, homeostatic model assessment-insulin resistance; NN, normal then normal intake; QUICKI, quantitative insulin sensitivity check index; $R C$, restricted then catch-up intake; $R N$, restricted then normal intake; $R R$, restricted then restricted intake.

Table 2. Measures of serum IGF-1, leptin, and adiponectin in the four groups

\begin{tabular}{lcccccc}
\hline Group & NN & RC & RN & RR & Model 1 (Pvalue) & Model 2 $(P$ value $)$ \\
\hline IGF-1 & $375 \pm 59$ & $380 \pm 61$ & $363 \pm 98$ & $324 \pm 67$ & $0.062^{\mathrm{a}}$ & $<0.0001^{\mathrm{b}}$ \\
Leptin & $2.91 \pm 1.00$ & $3.88 \pm 1.65$ & $2.11 \pm 0.93$ & $1.64 \pm 1.03$ & $<0.0001^{\mathrm{c}}$ & $<0.0001^{\mathrm{d}}$ \\
Adiponectin & $14.9 \pm 4.35$ & $14.4 \pm 3.20$ & $12.9 \pm 2.32$ & $14.1 \pm 2.96$ & $<0.0001^{\mathrm{e}}$ & $<0.0001^{\mathrm{f}}$ \\
\hline
\end{tabular}

IGF, insulin-like growth factor; NN, normal then normal intake; RC, restricted then catch-up intake; RN, restricted then normal intake; RR, restricted then restricted intake.

${ }^{a}$ Group effect $P=0.0308$, with RR being significantly less than either RC or NN. ${ }^{b}$ Weight was significantly positively associated with IGF-1 $(P<0.0001)$, and the group effect was not

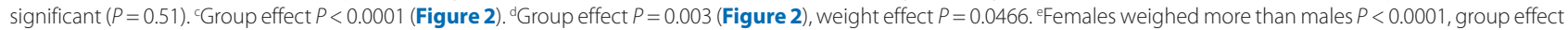
$P=0.38$. Females weighed more than males $P<0.0001$, group effect $P=0.0 .091$, weight effect $P=0.0066$.
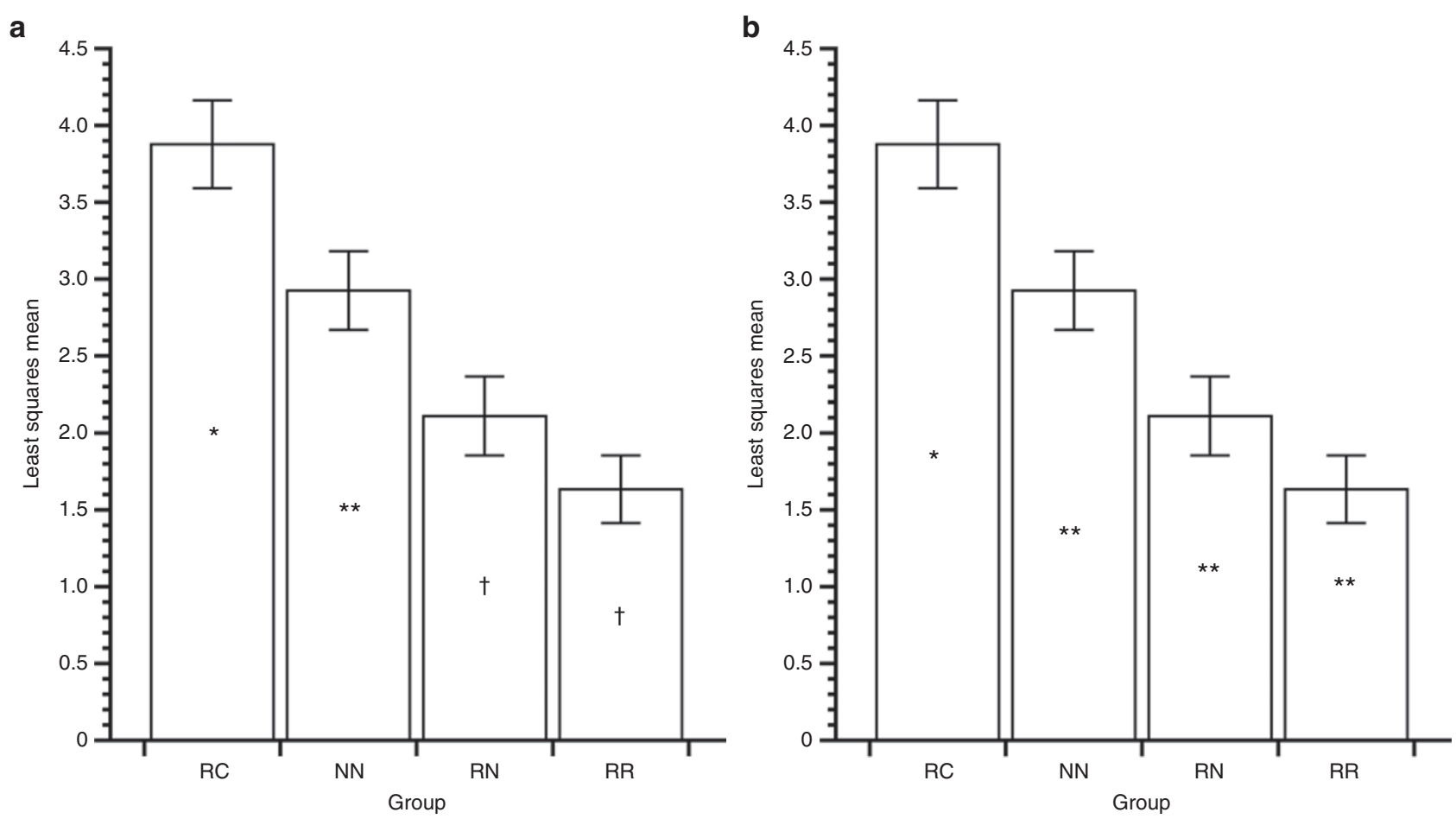

Figure 3. Least-squares mean serum leptin for the four study groups (a) accounting for gender and group (Model 1) and (b) accounting for gender, group, and current weight (Model 2 ). In each panel, bars not sharing the same symbol $\left(*, * *\right.$, or $\left.^{\dagger}\right)$ are significantly different. ${ }^{*}$ Significantly different from $\mathrm{RC}$, ** significantly different from RC and NN. NN, normal then normal intake; RC, restricted then catch-up intake; RN, restricted then normal intake; RR, restricted then restricted intake.

(Figure 3). Serum adiponectin was not significantly different among groups, but it was greater in females than in males $(P<0.0001)$.

\section{Passive Avoidance: Habituation}

$\mathrm{N}$ animals (for which early nutrition was normal $(\mathrm{NN})$ ) were more active than $\mathrm{R}$ animals (for which early nutrition was poor (RC, RR, or RN)), as evident from the significantly different incidences of timing out on day 20 . Only $5 \%$ of the $\mathrm{N}$ pups timed out as compared with $23.9 \%$ of the R pups $(P=$ 0.0033; Figure 4). The incidence of timing out on day 20 also differed significantly among the three R groups, with RN pups timing out significantly less often $(12.5 \%)$ than the RR $(25.0 \%$; $P=0.0378)$ or RC pups $(36.7 \% ; P=0.0350)$. 


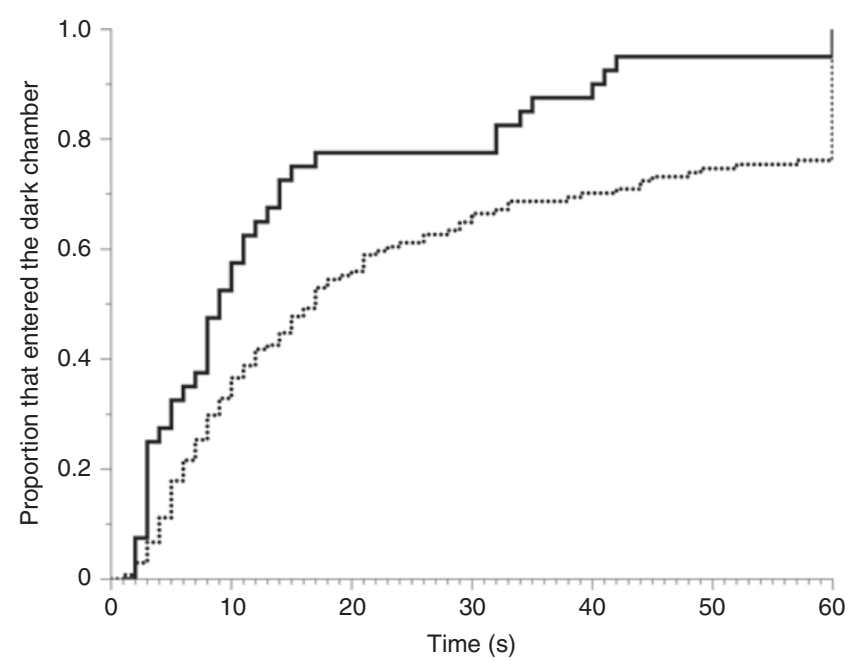

Figure 4. Kaplan-Meier survival analysis comparing the time taken for the NN (solid black line) and RN, RC, and RR animals combined (broken black line) to enter the dark chamber on day 21. The $y$-axis represents the proportion of animals that had entered the dark chamber, and the $x$-axis represents the time in seconds. NN, normal then normal intake; $\mathrm{RC}$, restricted then catch-up intake; $\mathrm{RN}$, restricted then normal intake; $R R$, restricted then restricted intake.

\section{Passive Avoidance: Learning}

On day 21, a similar number of $\mathrm{N}$ and $\mathrm{R}$ pups timed out by $60 \mathrm{~s}$, whether $(P=0.34)$ or not $(P=0.17)$ the presence of timing out on day 20 was accounted for. However, among the three $\mathrm{R}$ groups, $\mathrm{RC}$ pups were less likely to time out than $\mathrm{RR}$ $(P=0.0121)$ or RN $(P=0.0942)$ pups, although the last comparison was not statistically significant.

When timing out on day 21 was defined as a time $>120 \mathrm{~s}$, the data were little changed. Neither early diet group ( $\mathrm{N}$ vs. $\mathrm{R} ; P=0.56)$ nor gender $(P=0.36)$ was significant. However, among the $\mathrm{R}$ groups, there was a nonsignificant trend for the RC group to be less likely to time-out than the RR ( $P=$ $0.0633)$ or $\mathrm{RN}(P=0.0704)$ groups. This is consistent with the data using a cutoff of $60 \mathrm{~s}$ for "timing out" as described above.

\section{DISCUSSION}

Growth retardation in preterm infants is a predominantly postnatal event, so we aimed to develop a postnatal model of growth retardation in neonatal rats, using adjustments in litter size. Our results show that this is a simple, effective, and reproducible method of experimentally manipulating growth in rat pups. The pups and dams tolerated the manipulations well. In all cases, the cross-fostered pups were accepted by dams, and no pups were lost during the two periods of cross-fostering. This model differs from many existing models that have utilized prenatal interventions, or a combination of pre- and postnatal interventions. Few studies have compared the effects of prenatal and postnatal interventions, but those that have show that the two interventions are not equivalent. One study compared the effect of reduced maternal protein intake during pregnancy or during lactation $(9,10)$. Somatic growth was affected by the postnatal (lactation) intervention but not by the prenatal (pregnancy) intervention, whereas insulin sensitivity was affected by the prenatal but not the postnatal intervention (10).

Previous studies have demonstrated that manipulations in litter size are well tolerated by CD rats (11). They can lead to sustained changes in growth $(11,12)$ and in activation of the sympathetic nervous system (11), and, in some cases, changes in fasting insulin levels (12). In our model, early growth retardation (up to postnatal day 10) led to reduced somatic growth (by 20\%) and lower blood glucose. We were also able to identify significant differences in learning behavior from early growth retardation. Latency to enter the dark chamber of the passive avoidance apparatus was significantly greater in the growth-retarded animals on both the habituation and the testing day.

During the second intervention period, growth-retarded animals were randomized to one of three different litter sizes. The RC group showed rapid catch-up growth. Their growth was indistinguishable from that of the NN group by day 16 , and the two groups had almost identical growth thereafter. Although the NN and RC groups had similar body mass by the end of the study, their body composition was very different. The NN group had significantly lower body fat and significantly higher protein content. By contrast, the RN and NN groups had similar body composition despite their significant difference in body mass.

Not only did the RC group have a higher percentage of body fat, its serum leptin concentration was significantly greater than those of the other three groups even when body mass was accounted for. There were, however, no differences in IGF-1 (despite the between-group differences in lean mass) or in serum adiponectin levels. Previous rodent studies in which litter size has been manipulated have suggested that the effect on insulin, adiponectin, and leptin may vary depending on the time of assessment and on the postweaning diet (12). We were able to identify the effects of early growth retardation on serum IGF-1 and serum leptin and demonstrate that these changes were modifiable depending on the amount of subsequent catch-up growth. It is unclear how this relates to the preterm infant as long-term data on adiponectin, leptin, and IGF-1 are relatively limited. In one study, preterm infants had lower serum leptin than term infants (13) at $40 \mathrm{wk}$ of corrected age but not when body weight was accounted for. This is consistent with our results showing no between-group differences in serum adiponectin. However, comparison between our rodent data and existing human data is difficult, as the human data are often measured at birth or in early postnatal life, and typically do not assess the effects of catch-up growth on the biochemical outcomes.

Despite the differences in body mass, and in body composition, there were no significant differences in the measures of glucose homeostasis or insulin sensitivity/resistance (blood glucose, serum insulin, homeostatic model assessment-insulin resistance, homeostatic model assessment- $\beta$, and quantitative insulin sensitivity check index) among the groups. This is in agreement with the rodent data of Zambrago et al. (10), who showed that although prenatal nutritional interventions significantly affected 
insulin sensitivity, postnatal nutrient restriction did not, despite significant effects on somatic growth.

In our study, all four groups showed learning during the passive avoidance testing, but once differences in the habituation day were accounted for, the RC group showed more learned avoidance of the dark chamber than the RR or RN groups. Although the number of litters involved in the study was relatively small, and could confound the data, this finding would be consistent with human data (more normal growth rates in preterm infants being associated with better developmental outcome), and other rodent studies that have shown that early nutritional changes can have significant effects on brain structure (14) and on neurodevelopment or behavior (15).

There are several limitations to our study. It remains to be elucidated whether these differences in growth and body composition persist into later life, whether more sophisticated tests of insulin sensitivity will detect differences between the groups, and whether more detailed assessments of cognitive function, learning, and explorative behavior would confirm our findings. The relevance of animal data to humans is never certain, not least when considering preterm infants. Early effects of prematurity on metabolic risk and neurodevelopment in human preterm infants are likely modified by subsequent events that may either reduce the risk of adverse outcomes or increase it further. Moreover, we are not able to be certain that the betweenlitter differences are due to differences in nutrient intake or growth, as changes in litter sizes may have altered other factors (e.g., stress level of the pups or dam-pup interactions). Despite these limitations, we believe that this model is a promising way to study the effects of postnatal growth retardation and subsequent catch-up growth on growth, body composition, development, and insulin sensitivity. Moreover, this information may be relevant to the preterm human infant.

\section{METHODS}

\section{Animal Methods}

Pregnant CD rats (gestational day 14) were obtained from Charles River (Wilmington, MA). Rats were housed in plastic cages under constant conditions (temperature: $22^{\circ} \mathrm{C}$; humidity: $65 \%$ ) with a 12 -h dark:light cycle and were allowed to feed ad libitum. After a 7-d acclimatization period, litters were delivered and remained with the dam for $24 \mathrm{~h}$. On postnatal day 2, pups were randomly distributed among 7 litters of 10 pups each (N, normal intake) or 14 litters of 16 pups each $(\mathrm{R}$, restricted intake). On postnatal day 10, pups in the R group were randomly distributed among 5 litters of 6 pups each (RC, restricted then catch-up intake), 4 litters of 10 pups each (RN, restricted then normal intake), or 4 litters of 16 pups each (RR, restricted then restricted intake, $n=3$ litters). $\mathrm{N}$ pups continued in litters of 10 each $(\mathrm{NN}$, normal then normal intake, $n=5$ litters). Equal numbers of male and female pups were included in all the litters. Twenty $\mathrm{N}$ pups and $16 \mathrm{R}$ pups were killed on day 10 .

Pups were weighed on postnatal days 2, 5, 7, 10, 13, 16, 20, and 22. Passive avoidance testing was performed on postnatal days 20 and 21 (see below). Pups were killed on day 22, and tissues (including blood) were collected from half of the pups, whereas the remaining pups were stored at $-20^{\circ} \mathrm{C}$ pending whole-carcass body composition measurements.

The study was conducted under the auspices of Animal Resource Services of the University of California-Davis, which is accredited by the American Association for the Accreditation of Laboratory Animal Care. The University of California-Davis Institutional Animal Care and Use Committee approved the protocol.

\section{Timing of Nutritional Interventions}

Rats are more immature at birth than most other eutherian mammals (including humans). The dietary initial intervention in our study was carried out between birth and $10 \mathrm{~d}$ of postnatal age. This period is believed to be equivalent to third trimester in humans (16), although most recent systematic comparisons suggest that in terms of cerebral development, it is more likely representative of the early part of the third trimester (17). In either case, the early intervention is broadly equivalent to the period when reduced intake and poor growth are common in prematurely delivered human infants.

\section{Biochemical Analysis}

Two-third of the animals had biochemical testing $(\mathrm{NN}=20, \mathrm{RR}=$ $32, \mathrm{RN}=20$, and $\mathrm{RC}=16$ ) on day 22, after an overnight fast. Equal numbers of males and females were assessed, and representatives of all litters were included.

Serum insulin (Rat/Mouse Insulin ELISA Kit), c-peptide (Rat/ Mouse C-Peptide 2 ELISA), leptin (Rat Leptin ELISA Kit), and adiponectin (Rat Adiponectin ELISA Kit) concentrations were measured with their respective commercial ELISA kits (Millipore, St. Charles, MO). Serum IGF-1 concentrations were measured with a commercial kit (IGF-1 high-sensitive ELISA kit; IDS, Bolden UK). Blood glucose was measured in whole blood using a glucometer (EasyCheck; Home Aide Diagnostic, Deerfield Beach, FL). Homeostatic model assessment-insulin resistance and quantitative insulin sensitivity check index were calculated (18).

\section{Passive Avoidance}

Passive avoidance testing was carried out in all animals using an apparatus consisting of two Perspex boxes $(30 \mathrm{~cm} \times 20 \mathrm{~cm} \times 20 \mathrm{~cm})$ separated by a removable door. One of the boxes was black and wrapped in a lightproof cover (dark chamber), and the other was white and open to the light (light chamber). The chambers were separated by an opening with a removal door. The floor of the dark chamber was composed of a metal grid through which a small electrical charge could be passed. Testing was carried out over $2 \mathrm{~d}$ : a training (habituation) day and a testing (learning) day (19).

Habituation. On day 20, pups ( $n=30-48$ per group) were removed from their litter and placed at the center of the light chamber facing away from the door dividing the two chambers. After $30 \mathrm{~s}$, the divider between the chambers was removed, and the time taken for the rat to enter the dark chamber was recorded (i.e., once all four paws had touched the floor of the dark chamber). The removable door was replaced, separating the light and dark chambers. Thirty seconds later, a small electrical charge was applied to the floor of the cage for $2 \mathrm{~s}$. Ten seconds later, the rat was removed from the apparatus. If the rat did not enter the dark chamber within $60 \mathrm{~s}$, it was counted as having "timed out," and was picked up and placed in the dark chamber with the divider in place. Thirty seconds later, the shock was applied and the rat removed. The floor of the light and dark chambers was wiped clean with $5 \%$ bleach between each animal.

Testing. A similar procedure was carried out on the following day (day 21 ), except the subject was allowed $120 \mathrm{~s}$ to enter the dark chamber. No electrical shocks were applied on the second day.

Basis of the Test. Passive avoidance is a measure of learned avoidance. Rats are nocturnal and prefer the dark chamber. However, if they recall the painful stimulus associated with entering the dark chamber on day 20 , they would be expected to be less likely to enter it on day 21. An increased time to enter the dark chamber, or an increased likelihood of "timing out," between days 20 and 21, are taken as measures of learning.

\section{Body Composition}

One-third of animals had body composition assessed ( $N N=10, \mathrm{RR}$ $=16, \mathrm{RN}=10$, and $\mathrm{RC}=8$ ) on day 22 . Equal numbers of males and females were assessed, and representatives of all litters were included.

Body composition was assessed by carcass analysis. Water weight was calculated from change in weight before and after freeze-drying, fat 
weight from the change in weight after chloroform-methanol extraction, and ash weight following ashing in a muffle furnace $\left(600^{\circ} \mathrm{C}\right)(20)$.

\section{Statistical Analysis}

Weight data were analyzed by repeated-measures ANOVA with day of life, gender, and group as independent variables. Between days 2 and 10 of life, there were two different dietary groups ( $\mathrm{R}$ and $\mathrm{N})$, and between days 10 and 22, there were four dietary groups (NN, RC, RN, and RR).

Serum biochemistries were assessed using two-way ANOVA (with group and gender as independent variables) and by three-way ANOVA (with group, gender, and current weight as independent variables).

Testing of the effect of early growth restriction on passive avoidance was carried out using a two-stage procedure. First, differences between the $\mathrm{N}$ and $\mathrm{R}$ groups were assessed, then differences among the three $\mathrm{R}$ groups (RR, RN, and RC) were evaluated. Analysis was carried out by a combination of Kaplan-Meier analysis and nominal logistic regression. In the latter analysis, gender was also taken into account.

Analyses were carried out using JMP v7 (SAS Institute, Cary, NC). If two-way or three-way ANOVA suggested a significant group effect $(P$ $<0.1$ ), the possible effect of each individual independent variable was assessed using a Tukey post hoc test accounting for multiple testing. The independent variables were considered significant at $P<0.05$.

\section{STATEMENT OF FINANCIAL SUPPORT}

No financial assistance was received to support this study.

Disclosure: The authors declare no conflict of interest.

\section{REFERENCES}

1. Ehrenkranz RA, Younes N, Lemons JA, et al. Longitudinal growth of hospitalized very low birth weight infants. Pediatrics 1999;104(2 Pt 1): $280-9$.

2. Embleton NE, Pang N, Cooke RJ. Postnatal malnutrition and growth retardation: an inevitable consequence of current recommendations in preterm infants? Pediatrics 2001;107:270-3.

3. Cooke RJ. Postdischarge nutrition of preterm infants: more questions than answers. Nestle Nutr Workshop Ser Pediatr Program 2007;59:213-24; discussion 224-8.

4. Ehrenkranz RA, Dusick AM, Vohr BR, Wright LL, Wrage LA, Poole WK. Growth in the neonatal intensive care unit influences neurodevelopmental and growth outcomes of extremely low birth weight infants. Pediatrics 2006;117:1253-61.

5. Hack M, Breslau N, Fanaroff AA. Differential effects of intrauterine and postnatal brain growth failure in infants of very low birth weight. Am J Dis Child 1989;143:63-8.
6. Latal-Hajnal B, von Siebenthal K, Kovari H, Bucher HU, Largo RH. Postnatal growth in VLBW infants: significant association with neurodevelopmental outcome. J Pediatr 2003;143:163-70.

7. Wells JC, Chomtho S, Fewtrell M S. Programming of body composition by early growth and nutrition. Proc Nutr Soc 2007;66:423-34.

8. Singhal A, Fewtrell M, Cole TJ, Lucas A. Low nutrient intake and early growth for later insulin resistance in adolescents born preterm. Lancet 2003;361:1089-97.

9. Zambrano E, Bautista CJ, Deás M, et al. A low maternal protein diet during pregnancy and lactation has sex- and window of exposurespecific effects on offspring growth and food intake, glucose metabolism and serum leptin in the rat. J Physiol (Lond) 2006;571(Pt 1):221-30.

10. Zambrano E, Martínez-Samayoa PM, Bautista CJ, et al. Sex differences in transgenerational alterations of growth and metabolism in progeny (F2) of female offspring (F1) of rats fed a low protein diet during pregnancy and lactation. J Physiol (Lond) 2005;566(Pt 1):225-36.

11. Young JB. Effects of litter size on sympathetic activity in young adult rats. Am J Physiol Regul Integr Comp Physiol 2002;282:R1113-21.

12. Prior LJ, Velkoska E, Watts R, Cameron-Smith D, Morris MJ. Undernutrition during suckling in rats elevates plasma adiponectin and its receptor in skeletal muscle regardless of diet composition: a protective effect? Int J Obes (Lond) 2008;32:1585-94.

13. Siahanidou T, Mandyla H, Papassotiriou GP, Papassotiriou I, Chrousos G. Circulating levels of adiponectin in preterm infants. Arch Dis Child Fetal Neonatal Ed 2007;92:F286-90.

14. Brunette KE, Tran PV, Wobken JD, Carlson ES, Georgieff MK. Gestational and neonatal iron deficiency alters apical dendrite structure of CA1 pyramidal neurons in adult rat hippocampus. Dev Neurosci 2010;32:238-48.

15. Beard JL, Felt B, Schallert T, Burhans M, Connor JR, Georgieff MK. Moderate iron deficiency in infancy: biology and behavior in young rats. Behav Brain Res 2006;170:224-32.

16. Dobbing J, Sands J. Comparative aspects of the brain growth spurt. Early Hum Dev 1979;3:79-83.

17. Clancy B, Kersh B, Hyde J, Darlington RB, Anand KJ, Finlay BL. Webbased method for translating neurodevelopment from laboratory species to humans. Neuroinformatics 2007;5:79-94.

18. Heikkinen S, Argmann CA, Champy M-F, Auwerx J. Evaluation of glucose homeostasis. Curr Protocol Mol Biol 2007;2007:29B.3.1-B.3.2.

19. Tran TT, Chowanadisai W, Lönnerdal B, et al. Effects of neonatal dietary manganese exposure on brain dopamine levels and neurocognitive functions. Neurotoxicology 2002;23:645-51.

20. Warren LE, Horwitz BA, Hamilton JS, Fuller CA. Effects of $2 \mathrm{G}$ on adiposity, leptin, lipoprotein lipase, and uncoupling protein-1 in lean and obese Zucker rats. J Appl Physiol 2001;90:606-14. 